\title{
A Novel Method for Fabricating Microfluidic Devices Containing Immobilized Biological Specimens
}

\author{
Medina Hamidović ${ }^{*}$, Ferenc Ender ${ }^{2}$ \\ 1 Institute for Communications Engineering and RF-Systems, Johannes Kepler University Linz, \\ Altenbergerstraße 69, 4040 Linz, Austria \\ 2 Department of Electron Devices, Faculty of Electrical Engineering and Informatics, Budapest University \\ of Technology and Economics, H-1117 Budapest, P.O. Box 91, Hungary \\ * Corresponding author, e-mail: medina.hamidovic@jku.at
}

Received: 30 November 2018, Accepted: 30 January 2019, Published online: 28 March 2019

\begin{abstract}
Microfluidic devices are dominantly fabricated using the soft lithography microfabrication techniques and polydimethylsiloxane (PDMS) as a structural material. Although the technique is applicable for the majority of microfluidic devices, it has limited use for the fabrication of microfluidic devices with immobilized biological specimen due to the low biocompatibility- a consequence of the plasma-assisted bonding step during the assembly of the final device. In this step, biological specimens within the microfluidic device are affected by strong plasma exposure which ultimately can degrade their biochemical activity and stability. To the best of our knowledge, this paper presents for the first time a method for increasing the biocompatibility of a conventional PDMS soft lithography process and enables fabrication of the microfluidic devices containing immobilized biological specimens. Protection of the biological specimen during the plasma bonding step is ensured by placing a protective Polyvinyl Alcohol (PVA) nanofiber layer over the biological specimens. The method is verified against the conventional soft lithography method by fabricating microfluidic devices containing enzyme-filled microreactors and following enzymatic reactions. It was shown that inadvantageous impact of the plasma is reduced by utilizing a protective PVA layer which ultimately preserves the specific activity and biochemical stability of the immobilized enzymes. Keywords
\end{abstract}

microfabrication, polydimethylsiloxane (PDMS), polyvinyl alcohol nanofiber, immobilization, microfluidic devices

\section{Introduction}

Over the last two decades, the interest in utilizing microfluidic systems for various applications in biotechnology and biomedicine, mostly analysis and synthesis, has explosively grown. These microfluidic devices enable integrating numerous laboratory functions on a single chip that extends only few millimeters to centimetres in size $[1,2]$. These devices are suitably named Lab-on-a-chip $(L o C)$ devices as they enable scaling of multiple laboratory processes down to a single chip format [2]. In contrast to conventional batch processes, LoC devices can offer a number of compelling advantages, including, but not limited to, higher throughput and massive parallelism, improved efficiency, closed-system testing and better process control which reduces the potential of possible contamination and enables low response time and low reagent consumption [2, 3].

One of the key reasons for the popularization of LoC devices is their availability due to the ease of fabrication.
In fact, it was the development of the novel microfabrication techniques over the recent years that led to the presence of these microscope slide-sized laboratories in every modern biochemical laboratory [1-4]. The cost reduction and production time decrease is what allowed a large number of laboratories to transfer their research from a large scale (batch processing) down to the micro scale. Nowadays, microfluidic devices are fabricated at low costs, allowing for the rapid and massive production of cost-effective microfluidic chips [1-4].

Devices can be fabricated from diverse materials such as polymers (e.g. Polydimethylsiloxane (PDMS)), ceramics, semiconductors (e.g. silicon) and metals [5]. Choosing an optimal fabrication technique depends on the selected structural material and most common methods are soft lithography (dominantly used in combination with PDMS), metal deposition, etching, bonding, injection molding and 
embossing [5]. Accessing a large number of materials and methods makes it possible to design microfluidic chips with application-specific features such as optical, biological or chemical characteristics, which can then be rapidly prototyped at low production costs. The final choice of the material and the fabrication method depends on the targeted biochemical application.

In the field of microfluidics, the PDMS has become the material of choice for the microfabrication of the microfluidic devices due to its excellent physical properties [1]. The PDMS material offers good thermal and chemical stability- a crucial factor in biochemical applications, it is nontoxic, inert, biocompatible, non-fluorescent and optically transparent down to the wavelengths of $300 \mathrm{~nm}[1,2]$.

Although there are many methods for fabricating microfluidic devices in PDMS, the most common approach is the soft lithography method. Over the years, the method has become standardized and it can offer fast, easy and cost-effective fabrication of microfluidic PDMS devices. The process revolves around three main steps: mask developing, PDMS casting and bonding $[1,2,5]$. In the first step, a photosensitive resist and a light source are used to create a mold (a footprint) of the network of microfluidic channels and structures [1]. In the second step, PDMS is casted onto the mold and cured at high temperatures in order to imprint the microfluidic channels and features from the mold into the PDMS. After the curing, PDMS is released and cut from the mold leaving the imprinted channels and features open. During the final step, the PDMS is bonded to a substrate (usually glass or other PDMS slide) to seal the channels and form a final microfluidic device. In order to achieve bonding, both substrate and patterned PDMS are exposed to a strong oxygen plasma etching and brought into contact immediately after the exposure in order to form a permanent bond [2] (cf. Fig. 1).

Unfortunately, for the microfluidic applications that involve biological specimens, the bonding step of the soft lithography process represents a challenge since strong plasma exposure can effect the biochemical activity and stability of the specimen. This is the case when microfluidic devices that host immobilized biological specimens, often used in diagnostics [6,7], synthesis [8] or analysis [9], need to be fabricated. For example, in the case of microfluidic chips hosting immobilized enzymes, the enzyme stability is imperative for ensuring their high kinematic activity. When exposed to the plasma etching, the plasma can affect the stability, therefore leading to reduced enzymatic activity and efficiency. Here, a method

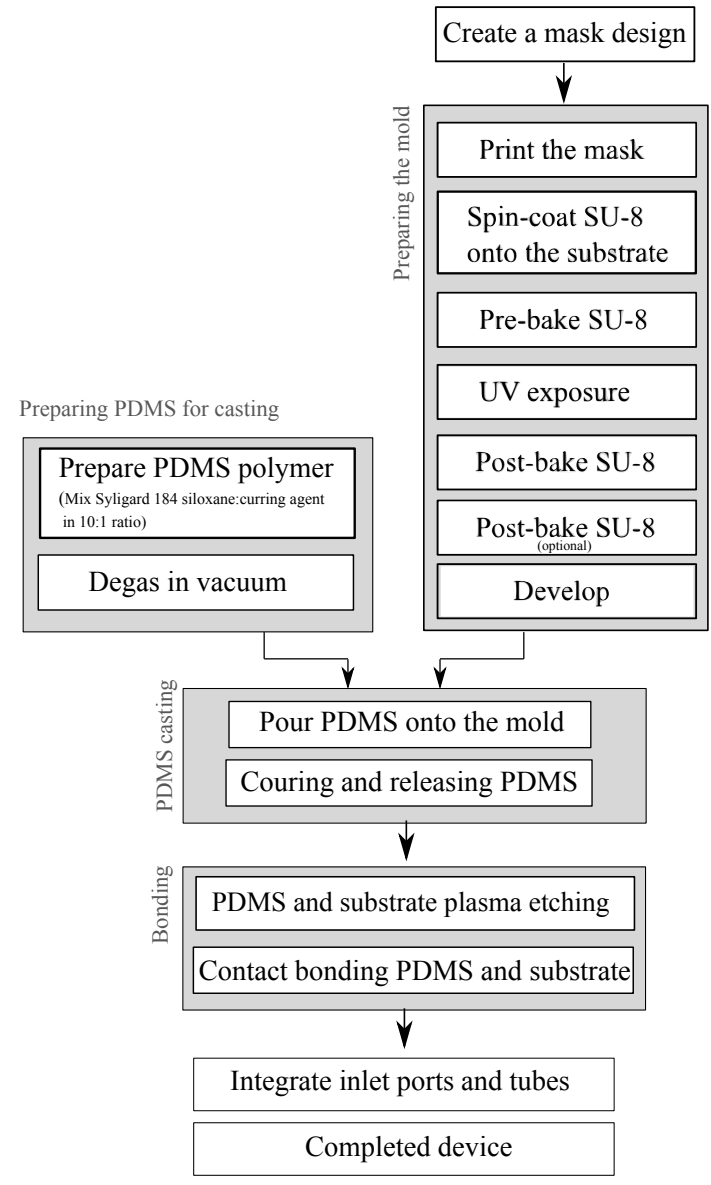

Fig. 1 The step-by-step process of soft lithography with three main steps: mask developing, PDMS casting and bonding.

to protect enzymes, or other biological specimens, during the plasma exposure is truly a necessity.

The scope of this work is to further investigate the destructive effects of the inevitable plasma exposure on the biological specimens, and to deliver a novel method that improves the applicability of the conventional soft lithography technique for fabricating microfluidic devices with immobilized specimens. We start by introducing the step-by-step conventional soft lithography process. In Section 3, we introduce microfluidic devices that host immobilized biological specimens, with a focus on LoC devices with immobilized enzymes. Section 4 gives a theoretical introduction of the novel fabrication technique, while Section 5 demonstrates the experimental validation of the proposed method. The final section compares and concludes the obtained results.

\section{Conventional Soft Lithography}

The standard soft lithography process technique is illustrated in Fig. 1. The process is usually carried out in the clean room in order to minimize the risk of any contamination, however 
the use of a clean room is not an absolute necessity except for the most demanding fabrication requirements [1, 2].

The process starts with designing the network of microfluidic channels and features. The design is printed and used to form a mold (a footprint) of the microfluidic channels and structures. Here, photosensitive resist (usually $\mathrm{SU}-8$ ) is spin-coated on the substrate (often Si wafer) in a controllable fashion depending on the desired thickness. The resist is then pre-baked in order to evaporate its solvent and lastly exposed to a light source (UV in the case of the SU-8 resist) to create a pattern based on the designed mask. Optional post-exposure baking steps aid in cross-linking the exposed portions of the SU-8 in preparation for its development. Lastly, SU-8 is developed leaving the finished pattern of microfluidic channels on the substrate for the use in casting of the PDMS.

Preparation of the PDMS chip is carried out as an independent step where curing agent and siloxane are mixed in order to prepare final PDMS polymer that is casted onto the fabricated SU-8 mold. Placing casted PDMS in an oven cures the PDMS, which can then be released from the mold and cut as desired.

In the last step, released PDMS and the cover substrate (usually glass or PDMS slide) are bonded using the oxygen plasma to form the final microfluidic device. Here, plasma exposure is used to aid the bonding by modifying the surface of both PDMS and the covering substrate. Untreated PDMS will not stick to any other substrate and it will be strongly hydrophobic [1]. For this reason, exposing PDMS to oxygen plasma reduces its hydrophobicity and aids bonding the PDMS to the glass substrate. To form a final microfluidic device, the two are brought into direct contact shortly after the plasma exposure (within the $60 \mathrm{~s}$ after the exposure) which forms a permanent bond and seals the device [1, 2].

Over the last two decades, the process illustrated in Fig. 1 has been a method of choice for fabricating various microfluidic devices that target numerous applications, both in continuous and two-phase microfluidics [10-12].

In these applications, the fabrication of the microfluidic device and experimental testing of the device are carried out as two independent steps (cf. Fig. 2(a)). In these applications, biological specimens are introduced only during the experimental/testing phase. It is only at that last phase that the specimens are delivered to the device through the microfluidic tubes, through the inlets of the device, and further processed on the chip.

However, for some microfluidic applications, fabrication and testing of the device cannot be carried out as two independent steps. This is the case for microfluidic devices where biological specimens are required to be permanently immobilized within the device [6-9]. This means that already at the fabrication phase, prior to assembling the final device, biological specimens are introduced into the device (cf. Fig. 2(b)). For these applications, conventional soft lithography process needs to be improved in order to protect immobilized specimens during the fabrication process.

In the following section, we briefly introduce concepts of microfluidic devices that host immobilized biological specimens in order to carry out chemical synthesis and analysis. Specifically, we focus on presenting microreactor LoC devices with immobilized enzymes [13-15].

\section{LoC devices with immobilized enzymes}

Compelling advantages of the microscale laboratory devices can be used to conduct and follow the biocatalytic (enzymatic) reactions in a more effective and controlled fashion then what conventional batch processing can offer $[12,13]$. In these applications, biological specimens of an interest are enzymes (biocatalysts) while chambers on the microfluidic chip where reactions occur are termed as microreactors [16].

\subsection{Enyzmes}

Enzymes are macromolecular biocatalysts that serve for accelerating chemical reactions [16, 17]. These catalysts are not only important for optimal functioning of all living beings but are also widely used in diverse sectors of bioprocess technology due to their green chemistry [17]. For this reason, various research studies on enzymatic reactions, more specifically enzymatic activity, productivity, stability, specificity and efficiency, have been proposed over the years [17-19].

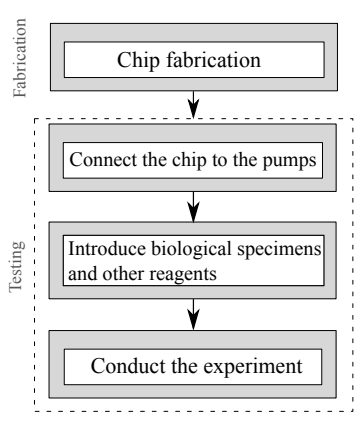

(a)

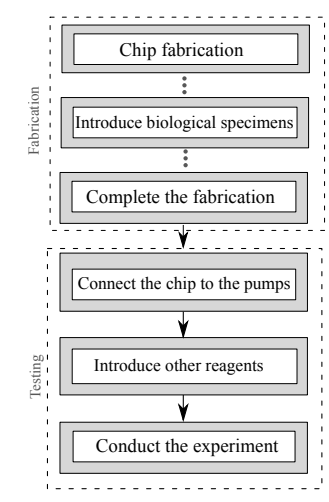

(b)
Fig. 2 Different stages of introducing the biological specimens into the device: (a) Specimens are introduced after the fabrication was completed;

(b) Specimens are introduced into the device during the fabrication. 
Enzymes can be free to move (free enzyme system) or they can be attached to a surface (immobilized enzyme system) [13-15]. Nowadays, immobilized enzymes are preferred over their free counterparts due to the increased enzymatic stability over a considerably long period of time [17]. Immobilization of enzymes is a confinement of an enzyme to some sort of a biocompatible surface such as beads, gels, nanopores, magnetic nanoparticles, and recently, polymer nanofibers [13-15, 17]. Immobilization allows for enzymes to be held in place throughout the biochemical reaction, following which they are easily separated from the products and may be re-used for another set of biochemical reactions, therefore enabling enzyme recycling.

Although various techniques were demonstrated successful for enzyme immobilization, entrapment or caging of enzymes by covalent or noncovalent bonds within gels or electrospun nanofibers have truly revolutionized the world of enzyme immobilization. This is due to the wide applicability of the entrapping methods in the fields of chemistry, biomedicine biosensors and biofuels [17]. Moreover, a broad spectrum of structural materials available to construct the nanofibers makes the method suitable for various microfluidic applications. Fig. 3 shows SEM images of an enzyme immobilization within nanofibers of different diameters as a result of different electrospinning settings (see Section 4.3).

\subsection{Enyzmatic microreactors}

Microreactors are defined as miniaturized reaction systems that are often employed in biocatalytic applications where due to the small sizes enzymatic processes are more effective and economical [19]. In contrast to conventional batch methods of following the biocatalyctic reactions, LoC devices with microreactors offer accelerated process

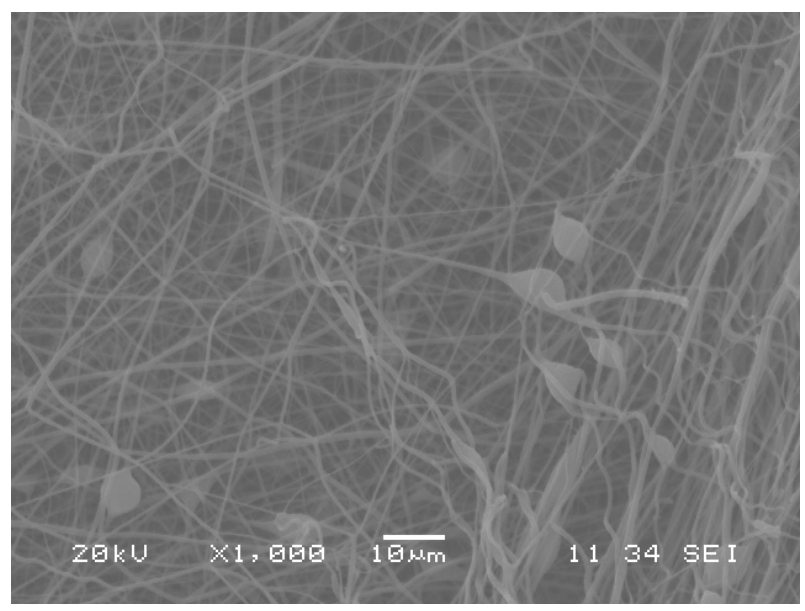

development, enhanced safety, stable and controlled temperature distribution inside the reactor volumes, short reaction times and high throughput [19]. Moreover, microreactors are characterized by an outstanding repeatability and reproducibility- a key features in employing these structures in investigating enzymatic kinetics.

In biocatalytic applications, the efficiency of the microreactor can be further improved by immobilization of enzymes inside the microreactors. This offers a high degree of re-usability of the biocatalyst which results with more economical and more environmentally friendly process [17, 19]. Eventually, integrating immobilized enzymes onto the LoC devices can offer a high throughput screening of enzymes and their substrates. This could lead to a wide range of potential applications of microreactors in biotechnology, as well as combinatorial chemistry and enzyme targeted drug search [17, 19].

Despite the rapid development of enzymatic microreactors in the recent decade, important design and fabrication questions still remain unanswered, therefore limiting the possibilities of the research in this field [19]. Moreover, this results with the lack of the experimental validation of the already existing enzymatic microreactor models. In order to offer possibilities for further advancements in protein and enzyme research, the next section introduces a novel method for adopting conventional soft lithography process for the fabrication of LoC devices with microreactors that host immobilized enzymes.

\section{Novel fabrication method}

Standard microfluidic chip is usually fabricated using the PDMS as a structural material and the soft lithography process as a fabrication technique, due to their easiness of use and fast prototyping [2]. It is then somewhat

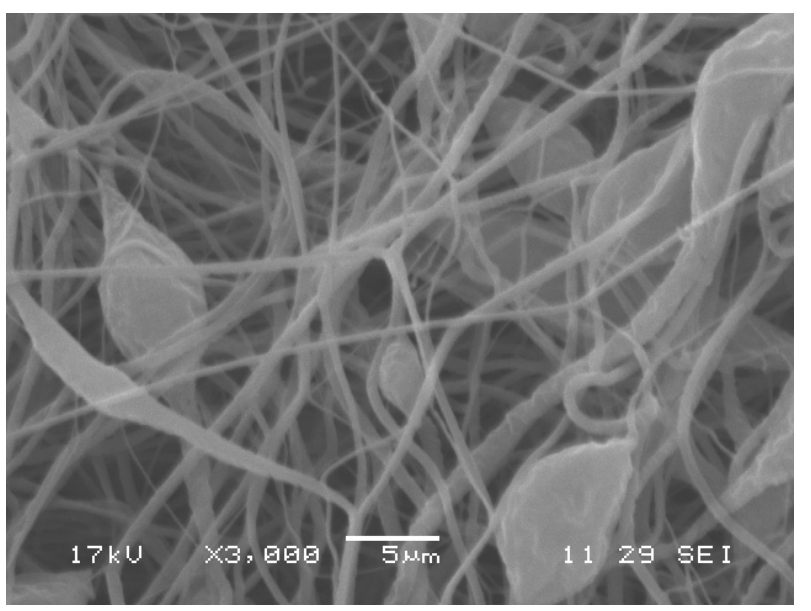

Fig. 3 Scanning Electron Microscopy (SEM) analysis of Lipase CaLB enzyme entrapped within the PLA nanofibers ranging from 200 nm to 700 nm. 
expected to use the availability of this conventional process and rather than delivering a completely new fabrication method, to focus on finding a way to adopt the already standardized process for the fabrication of microfluidic device with biological specimen.

We start by introducing the general workflow of the novel fabrication technique, as shown on Fig. 4, followed by more details on the materials and methods of sub-steps of the proposed technique.

\subsection{The general working principle}

The proposed microfluidic device has two important parts: glass substrate holding the biological specimens (enzymes, proteins or cells) and a PDMS chip with imprinted microchannels and microreactors. For this reason, the fabrication process is divided into two independent parts which can be performed in parallel, thus leading to even faster device prototyping.

The complete fabrication process is shown in Fig. 4. The left part of the figure demonstrates a typical PDMS molding and casting process, used to fabricate a network of channels and microreactors inside the PDMS material. The right side of the figure demonstrates the multistep fabrication of the glass substrate with immobilized enzymes ${ }^{1}$. In the first step, an enzyme-enriched Polylactic acid (PLA) polymer solution is electrospun onto the clean glass substrate, thus forming a layer of nanofibers with immobilized enzymes. This layer is the layer that provides the enzymatic activity for the microfluidic chip. In the following step, laser engraving (or other patterning technique) is used to pattern the design of a microreactor area on the electrospun nanofiber layer. Afterwords, a second layer of the Polyvinyl Alcohol (PVA) nanofibers is deposited on top of the first layer. The PVA layer does not hold immobilized enzymes and serves solely to protect the underlying layer of PLA nanofibers during the forthcoming plasma treatment. To make the design of the two layers of nanofibers compatible, the design of a microreactor area is again engraved in the PVA layer in the same manner as previously for the PLA layer.

To construct the final microfluidic device, the PDMS chip and the glass substrate with the nanofibers are exposed to plasma. Bringing the two parts into direct contact after the exposure, the final device is assembled and fabrication process is completed.

$\mathbf{1}$ The process can be applied for fabricating glass substrates with other immobilized biological specimens such as proteins or cells.

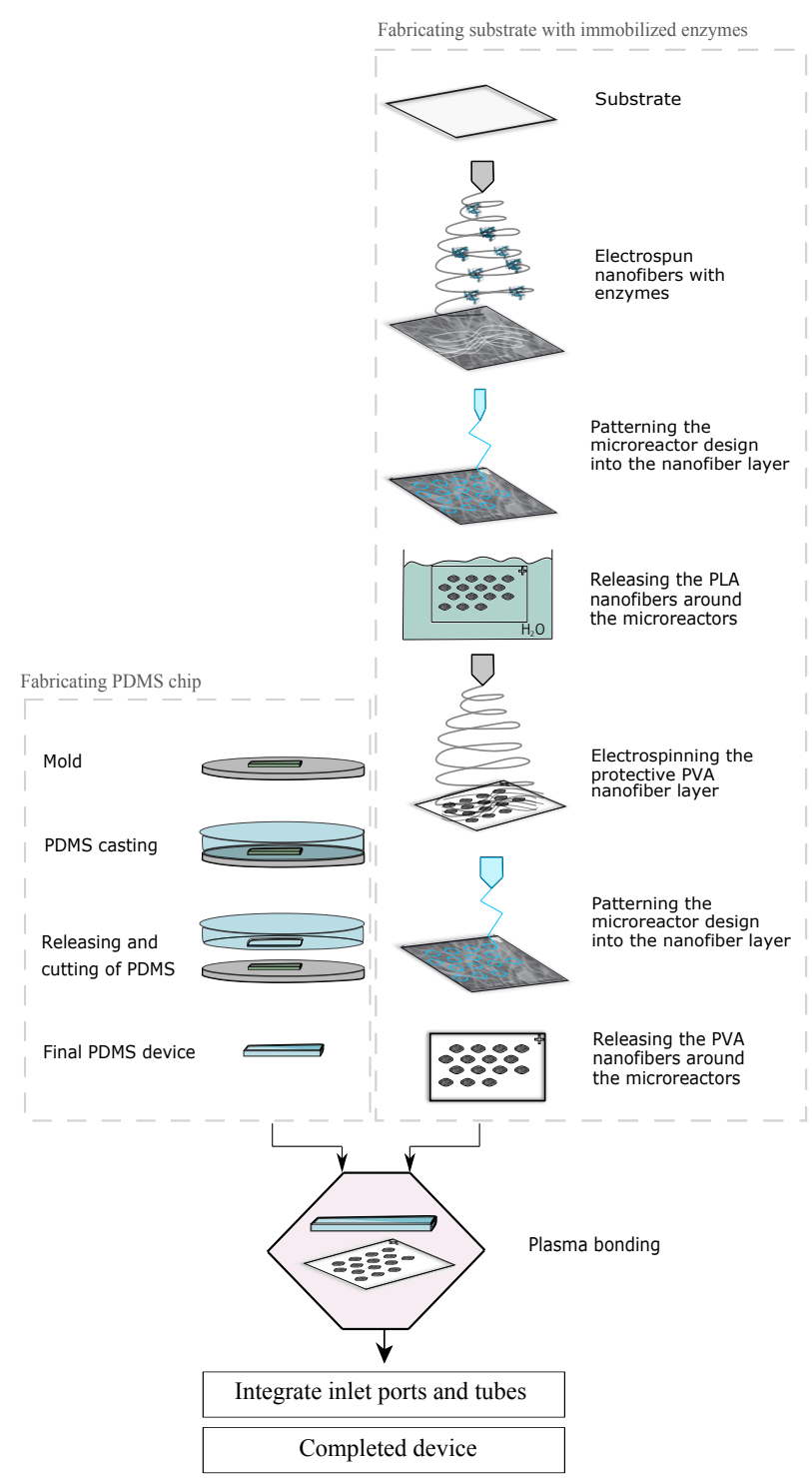

Fig. 4 A novel method for fabricating microfluidic microreactor devices with immobilized enzymes. The process is divided into two independent steps: (left) conventional PDMS molding and casting for fabricating PDMS chip; (right) fabricating glass substrate that holds immobilized enzymes.

\subsection{Fabricating the PDMS chip}

To fabricate the PDMS chip, the siloxane oligomer and cross-linker/curing agent (Sigma-Aldrich, Sylgard 184) are thoroughly mixed at a ratio of 10:1 by weight and afterwards degassed in a vacuum chamber. The PDMS pre-polymer is poured/casted over the silicon master mold that was SU-8 patterned using the conventional photolithography. Casting the PDMS over the mold, the PDMS conforms to the shape of the master and replicates its features with a high resolution $[1,2]$. The curing or cross-linking of the PDMS took place at room temperature over the period of 24 hours. As an alternative, the curing can be 
accelerated by enhancing the temperature e.g. $\left(70{ }^{\circ} \mathrm{C}\right.$ for $60 \mathrm{~min}$ ). The PDMS can then be peeled off from the mold leaving the final PDMS chip.

\subsection{Fabricating the glass substrate with the enzyme}

A microscope glass slide (1mm in thickness), used as a substrate for the nanofibers, was carefully cleaned using an IPA (Isopropyl alcohol) and a clean-room tissue to remove the dust. In this step, the minute amount of IPA applied to the layers via the tissue evaporates in a few seconds after the application and does not affect the following process. To fabricate nanofibers with immobilized enzymes, electrospinning as the most common, simple and versatile process for the production of fine fibers is used [20]. Using the electrostatic field, the technique allows for the production of long, three-dimensional, ultrafine fibers with diameters in the range of a few micrometres to nanometres [13]. For an enzyme entrapment within the nanofibers, a freshly prepared CaLB as lyophilized enzyme powder was added to the PLA polymer to form a $10 \%$ enzyme-polymer mixture. The resulting mixture was agitated in an ultrasound bath for $25 \mathrm{~min}$ and then electrospun onto the glass substrate. Electrospinning experiments were performed at room temperature $(24 \pm 2 \mathrm{C})$ using a specially designed in-house tool. By varying parameters, such as the applied voltage $(10-30 \mathrm{kV})$ and the distance from the polymer needle to the glass substrate mounted on the collector plate it is possible to generate nanofibers of varying parameters, as previously shown in Fig. 3 .

In order to engrave the layouts of the microreactors onto the nanofiber layer, laser cutting is used. In this work, solid-state, $355 \mathrm{~nm}$ Nd:YAG (neodymiumdoped yttrium aluminium garnet) laser is used as it offers controllable output power and absolute precision of $1 \mu \mathrm{m}$ which allows for precise layout patterning. Moreover, the controllable output power makes this laser more suitable for the use with the thin substrates and with the biological specimens, since the damage caused by the heat transfer can be minimized, thus making the engraving process more efficient and more stable.

To protect the layer of nanofibers with the immobilized CaLB enzymes, a sacrificial layer of PVA nanofibers is electrospun on top of the first PLA enzyme-enriched layer. The PVA is a non-toxic, water-soluble synthetic polymer with a high degree of resistance for the plasma ${ }^{2}$.

2 Behavior of the PVA material under the plasma exposure was observed during the number of experimental trials.
The PLA deposition is a critical step for enabling further fabrication and ensuring a correct functionality of the device. During the electrospinning of PVA, it is necessary to assure that the underlying PLA layer with enyzmes is completely covered with the top PVA layer. Graphical illustration of the protective PVA layer is shown in Fig. 5.

In the following step, the PVA layer is laser patterned using the same microreactor design that was used to pattern the PLA layer. An example of a microfluidic chip with two layers of laser engraved nanofibers is shown in Fig. 6.

\subsection{Plasma bonding}

In the final step, the microfluidic chip is assembled by exposing the glass substrate with nanofibers, together with the PDMS chip, to oxygen plasma for 30 seconds. Within the 60 seconds after the exposure, the two parts are brought into direct contact to form a permanent bond and seal the device. The strong strength of the plasma-assisted bonding ensures that no leaking will occur during the testing of the device and provides the opportunity to re-use the device for multiple experiments.

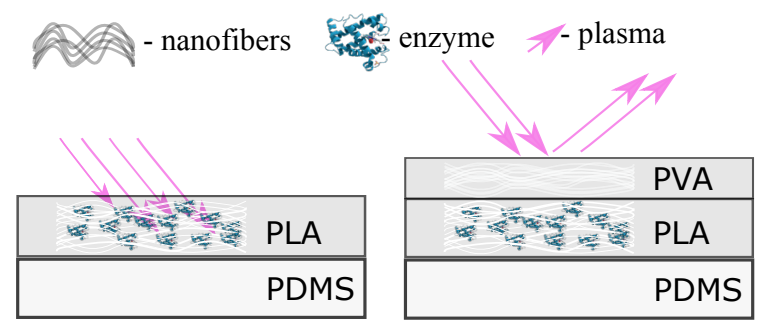

Fig. 5 Exposing PLA and PVA to plasma: (left) having no protection on top of the PLA nanofiber layer with enyzmes, the plasma can penetrate through the fibers and effect the stability of the immobilized enzymes; (right) having high resistance layer of PVA nanofibers on top of the

PLA layer protects the enzymes during the plasma exposure.

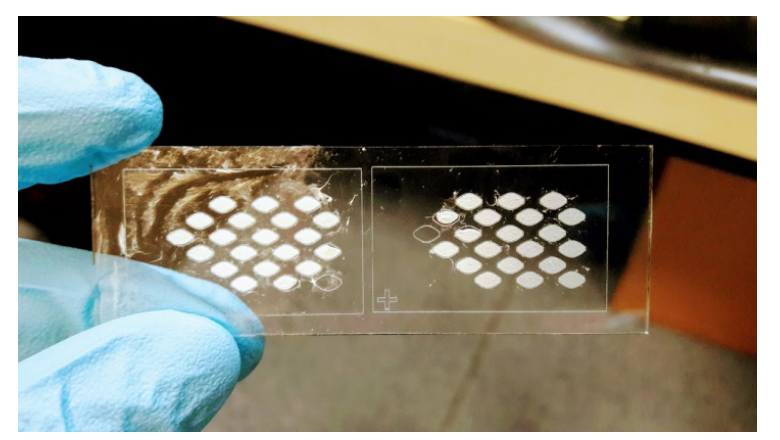

Fig. 6 An example of laser engraved microreactor layout on two layers of nanofibers: PLA layer with immobilized enzymes as a first layer, followed with the protective PVA layer. 


\section{Experimental validation}

To validate that the proposed method can truly be used in fabricating LoC devices with biological specimens, we have fabricated a microfluidic device with enzyme-filled microreactors according to the design shown in Fig. 6. Before presenting the results collected from the biocatalytic measurements, we give a brief background on the principle of microreactors and enzymatic activity.

\subsection{The principle of the microreactor stage}

In this work, the reactor stage is designed in a form of cylindrical chambers (hollows) inside the PDMS material, whose content are nanofibers with immobilized enzymes.

When reactor holds supporting materials such as nanofibers, and biocatalyst is immobilized onto their surfaces, it is defined as the packed-bed reactor [22]. As shown in Fig. 7 , in the basic working principle, the reaction fluid enters the packed bed reactor, the reaction with biocatalyst develops in the microreactor stage, and outlet fluid is collected at the end of the microreactor. The target molecules of the enzymes dissolved in the reaction fluid is known as their substrate, and it is selectively bonded to the binding site of the enzyme that further catalyses the transformation of the substrate to a product [15]. In this work, an enzyme CaLB catalyses the transformation of the substrate $\mathrm{p}$-Nitrophenyl palmitate (pNPP) to the product $\mathrm{p}$-nitrophenol ( $\mathrm{pNP}$ ). The enzymatic reaction takes place inside the microreactor area, as shown in Fig. 7.

\subsection{Materials and Methods}

\subsubsection{Microreactor specifications}

After electrospinning the nanofibers with immoblized CaLB enzyme, the electrospun mass is measured to be $3060 \mathrm{mg}$ (3000 mg of PLA solution and $60 \mathrm{mg}$ enzyme).

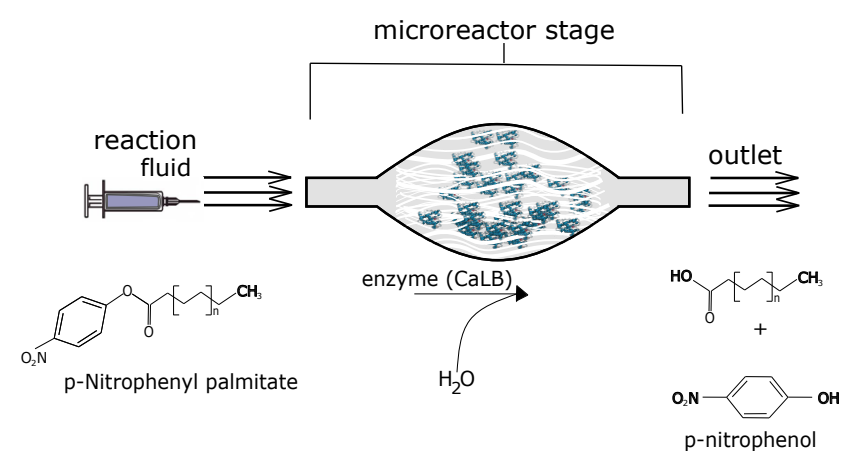

Fig. 7 Packed-bed reactor: the enzyme-substrate reaction between the immobilized enzyme CaLB and the reaction fluid p-Nitrophenyl palmitate takes place inside the microreactor stage.
Considering that PLA solution is $10 \% \mathrm{w} / \mathrm{w}$ solution, this implies that total chip holds $300 \mathrm{mg}$ of PLA polymer. Therefore, at the end of electrospinning process the chip hosts $300 \mathrm{mg}$ of PLA and $60 \mathrm{mg}$ of CaLB enzyme, which means that enzyme/polymer ratio on the chip is $60 / 300=20 \%$. In average, it was measured that every electrospun chip carries 2-3 mg of CaLB enzyme. Moreover, the complete microreactor area chamber is measured to be as $A_{\text {chamber }}=21 \times 4,635 \mathrm{~mm}^{2}=97.33 \mathrm{~mm}^{2}$, while the total chip has the area of $A_{\text {chip }}=650 \mathrm{~mm}^{2}$. This implies that microreactor chamber area is $15 \%$ of the total chip area. This further implies that the $15 \%$ of total $2-3 \mathrm{mg}$ of electrospun CaLB enzyme is contained in the microreactor. Finally, this gives the approximation of the amount of CaLB enzyme in every chamber of $15 \mu \mathrm{g}$, or in total microreactor stage of $0.32 \mathrm{mg}$.

\subsubsection{Materials and tools}

PLA (D-isomer Polylactic Acid $\backslash 10 \%$, PURASORB PL 24) was obtained from PURAC (Gorinchem, Netherlands). Solvents (chloroform and ethanol) were obtained from Molar Chemicals (Budapest, Hungary). N,NDimethylformamide (DMF) was obtained from Merck (Budapest, Hungary). PLA solution is $10 \% \mathrm{~m} / \mathrm{m}(9 \%$ polymer and $91 \%$ solvent). CaLB as lyophilized enzyme powder (recombinant C. antarctica lipase B) was obtained from c-LEcta (Leipzig, Germany). For an enzyme entrapment within the nanofibers, the PLA solution (PLA solved in chloroform and dimethylformamide in 6/1 ratio) with CaLB as lyophilized enzyme powder was prepared where $1 \mathrm{~g}$ of PLA solution contains 10mg of CaLB enzyme powder. Since PLA polymer solution is $10 \%$ polymer and $90 \%$ solvent, the final polymer emulsion contains $10 \%$ enzyme. Silicon Elastomer kit for fabricating PDMS chip was obtained from Sigma-Aldrich (Saint Louis MO, USA). To pattern the nanofibers, an Nd:YAG (AVIA Coherent, USA) laser was used. For the purpose of plasma treatment, Femto plasma cleaner (Diener electronics, Germany) was used. To follow the enzyme kinetics, gaschromatography(GC) on an Agilent 4890 equipment using Hydrodex b-6TBDM column (Machery-Nagel, $25 \mathrm{~m} \times 25 \mathrm{~mm} \times 0.25 \mu \mathrm{m}$, heptakis(2,3-di-Omethyl-6-O-t-butyldimethylsilyl)-b-yclodextrin) was used. Protective PVA solution was prepared with aqueous solvent (675 mg: $10 \mathrm{~m} / \mathrm{m} \%$ PVA in distilled water).

\subsubsection{The substrate-product transformation}

The assay was performed by measuring the increase in the absorbance expected at around $350-400 \mathrm{~nm}$ produced by 
Table 1 Catalytic behavior of the CaLB enyzmes for different fabrication methods: Polymer type PLA specifies that enzyme-enriched layer of PLA nanofibers was directly exposed to the plasma, while PLA+PVA corresponds to the novel fabrication method. By comparing the values of the enzymatic parameters, it can be noticed that protective PVA layer contributes to higher catalyctic activity of the immobilized enzymes.

\begin{tabular}{lcccccc}
\hline $\begin{array}{l}\text { Polymer } \\
\text { type }\end{array}$ & $\begin{array}{c}\text { Plasma } \\
\text { time }(\mathrm{s})\end{array}$ & $\begin{array}{c}\mathrm{RT}^{3} \\
(\mathrm{~h})\end{array}$ & $\begin{array}{c}\mathrm{c} \\
(\%)\end{array}$ & ee $(\%)$ & $\mathrm{E}$ & $\mathrm{U}_{\mathrm{B}}$ \\
\hline $\begin{array}{l}\text { PLA } \\
\text { PLA+ }\end{array}$ & 12 & 2 & 0.8 & 77.8 & 8.1 & 2.6 \\
PVA & 12 & 2 & 1.6 & 91.1 & 22.1 & 5.3 \\
PLA & 12 & 2 & 0.7 & 92.5 & 26.2 & 2.8 \\
$\begin{array}{l}\text { PLA+ } \\
\text { PVA }\end{array}$ & 12 & 2 & 0.9 & 92.3 & 25.3 & 3.0 \\
PLA & 12 & 4 & 2.0 & 97.6 & 89.5 & 3.3 \\
$\begin{array}{l}\text { PLA }+ \\
\text { PVA }\end{array}$ & 12 & 4 & 4.3 & 98.1 & 100 & 7.2 \\
PLA & 12 & 4 & 1.5 & 96 & 51.3 & 3 \\
$\begin{array}{l}\text { PLA+ } \\
\text { PVA }\end{array}$ & 12 & 4 & 1.8 & 97.2 & 71.8 & 3 \\
\hline
\end{tabular}

the release of a product $p$ - nitrophenol in the hydrolysis of $0.4 \mathrm{mM}$ pNPP (MW $=377: 52 \mathrm{~g} / \mathrm{mol}$ ) in a Phosphatebuffered saline (PBS) buffer $(\mathrm{m}=0.1 \mathrm{M}, \mathrm{pH}=7.5)$ at the temperature of $30^{\circ} \mathrm{C}$. To make a final reaction fluid, $7.8 \mathrm{mg}$ of pNPP was solved in $20 \mathrm{ml}$ PBS buffer. This stock solution was mixed for 27 hours to be diluted completely for the on-chip reaction. Reaction fluid enters the chip over the inlets with input flow rate in the range of $1-5 \mu \mathrm{L} / \mathrm{min}$. The substrate-enzyme reaction is carried out in situ, and the product is collected at the output.

\subsection{Measurements}

For following the enzyme kinetics (parameters that describe the rate of substrate-product transformation) we observed the conversion rate (c), enantiomeric excess (ee), enantiomeric ratio (E), and specific activity $\left(U_{B}\right)$ of an enyzme, as a measurement of an enzyme purity and reaction rates. The measurements were obtained using the gas spectroscopy (GC) tool.

Table 1 gives the values of the kinetic parameters for the two types of measurmenets: i) the measurements carried out when enzyme-enriched PLA nanofibers were directly exposed to the plasma (conventional fabrication process) and ii) the measurements carried out when protective PVA layer is electrospun on top of the enzyme-enriched PLA layer (the novel proposed method). As it can be seen in Table 1, a significant increase in all reaction parameters can be noticed when enzymes are protected by the PVA nanofiber layer. The values obtained demonstrate the importance of utilizing the protective PVA layer to protect the underlaying enzymes. Moreover, two sets of tests were carried out for the same conditions to further verify the obtained results. As it can be seen in Table 1 (row 2 and 4, as well as row 6 and 8) similar results are obtained for these tests (small deviations in presented results can be reasoned by possible ununiformity of plasma in the chamber as well as the change of the room temperature while conducting experiments).

\section{Conclusion}

In this work, we have proposed a novel technique for fabricating biocompatible microfluidic chips that host immobilized biological speciments. The technique was optimized for the purpose of fabricating LoC devices that host nanofiber-immobilized enzymes inside the microreactor stage. The proposed method solves the great challenge of the conventional fabrication technique by means of protecting the immobilized enzymes from the plasma treatment which was a true necessity. Here, a novel approach utilizing protective PVA layer on top of an enzyme-enriched PLA layer, was introduced and experimentally verified. Results demonstrated that the PVA layer can successfully protect the enzymes during the plasma treatment and therefore ensure their higher enzyme activity for a later use.

\section{Acknowledgement}

Authors are deeply thankful to Dr. Tamás Hurtony, at the Department of Electronics Technology, for his help and advice on the laser fabrication technology. Authors would also like thank to Dr. Kolos Molnar, at the Department of Polymer Technology, for providing high power supply for the electrospinning, and Dr. Diana Weiser, Department of Organic Chemistry and Technology, for her assistance on the project and for providing materials and resources needed for the experiments. 


\section{References}

[1] Friend, J., Yeo, L. "Fabrication of microfluidic devices using polydimethylsiloxane", Biomicrofluidics, 4(2), Article ID: 026502, 2010 .

https://doi.org/10.1063/1.3259624

[2] Dzebic, M., Kurikov, O., Dobroliubov, O., Nava, O. C. A. "Design and Fabrication of a PDMS Microfluidic Device for Titration of Biological Solutions", In: Badnjevic, A. (ed.) CMBEBIH 2017, IFMBE Proceedings, Vol. 62, Springer, Singapore, 2017, pp. $147-152$.

https://doi.org/10.1007/978-981-10-4166-2_23

[3] Saikat, C., Sharath, M., Srujana, M., Narayan, K., Kumar, P. P. "Modelling and analysis of microfluidic micromixer for Lab-ona-Chip(LoC) application", In: Annual IEEE India Conference Proceedings (INDICON), New Delhi, India, 2015, pp. 1-6. https://doi.org/10.1109/INDICON.2015.7443325

[4] Iliescu, C., Taylor, H., Avram, M., Miao, J., Franssila, S. "A practical guide for the fabrication of microfluidic devices using glass and silicon", Biomicrofluidics, 6(1), ID: 016505-016505-16, 2012. https://doi.org/10.1063/1.3689939

[5] Leester-Schadel, M., Lorenz, T., Jürgens, F., Richter, C. "Fabrication of Microfludic Devices", In: Dietzel, A. (ed.) Microsystems for Pharmatechnology, Manipulation of Fluids, Particles, Droplets and Cells Springer, Cham, Switzerland, 2016, pp. 23-57.

https://doi.org/10.1007/978-3-319-26920-7

[6] Wang, M. S., Black, J. C., Knowles, M. K., Reed, S.- M. "C-reactive protein (CRP) aptamer binds to monomeric but not pentameric form of CRP", Analytical and Bioanalytical Chemistry, 401(4), pp. 1309-1318, 2011. https://doi.org/10.1007/s00216-011-5174-1

[7] Song, Y. S., Shin, H. Y., Lee, J. Y., Park, C., Kim S. W. "b-Galactosidase-immobilised microreactor fabricated using a novel technique for enzyme immobilisation and its application for continuous synthesis of lactulose", Food Chemistry, 133(3), pp. 611-617, 2012 .

https://doi.org/10.1016/j.foodchem.2012.01.096

[8] Gijs, M. a. M., Lehmann, U. "Microfluidic Applications of Magnetic Particles for Biological Analysis and Catalysis", Chemical Reviews, 110(3), pp. 1518-1563, 2010. https://doi.org/10.1021/cr9001929

[9] Churski, K., Kaminski, T. S., Jakiela, S., Kamysz, W., BaranskaRybak, W., Weibel D. B., Garstecki, P. "High-throughput automated droplet microfluidic system for screening of reaction conditions", Lab on a Chip, 12, pp. 1629-1637, 2012. https://doi.org/10.1039/B925500A

[10] Boedicker, J. Q., Li, L., Kline, T. R., Ismagilov, R. F. "Detecting bacteria and determining their susceptibility to antibiotics by stochastic confinement in nanoliter droplets using plug-based microfluidics", Lab on a Chip, 8, pp. 1265-1272, 2008. https://doi.org/10.1039/b804911d
[11] Han, S. J., Park, H.-K., Kim, K. S. "Applications of Microfluidic Devices for Urology", International Neurourology Journal, 21(Suppl 1), pp. S4-9, 2017. https://doi.org/10.5213/inj.1734838.419

[12] Ender, F., Weiser, D., Nagy, B., Bencze, L. Cs., Paizs, Cs., Pálovics, P., Poppe, L. "Microfluidic multiple cell chip reactor filled with enzyme-coated magnetic nanoparticles - An efficient and flexible novel tool for enzyme catalyzed biotransformation", Journal of Flow Chemistry, 6(1), pp. 43-52, 2016. https://doi.org/10.1556/1846.2015.00036

[13] Ender, F., Weiser, D., Vitéz, A., Sallai, G., Németh, M., Poppe, L. "In-situ measurement of magnetic nanoparticle quantity in a microfluidic device", Microsystem Technologies, 23(9), pp. 39793990, 2015. https://doi.org/10.1007/s00542-015-2749-3

[14] Ender, F., Németh, M., Pálovics, P., Drozdy, A., Poppe, A. "Thermal compact modeling approach of droplet microreactor based Lab-on-a-Chip devices", Microelectronics Journal, 45(12), pp. 1786-1794, 2014. https://doi.org/10.1016/j.mejo.2014.07.005

[15] Bisswanger, H. "Chapter 2: Enzyme Kinetics", In: Enzyme Kinetics, Principles and Methods, Second Edition, 2nd ed., WileyVCH, Weinheim, Germany, 2008, pp. 124-143. https://doi.org/10.1002/9783527622023

[16] Sarrouh, B., Santos, T. M., Miyoshi, A., Dias, R., Azevedo, V., "Up-To-Date Insight on Industrial Enzymes Applications and Global Market", Journal of Bioprocessing \& Biotechniques, ID: S4:002, 2012 https://doi.org/10.4172/2155-9821.S4-002

[17] Bartsch S., Bornscheuer, U. T. "A Single Residue Influences the Reaction Mechanism of Ammonia Lyases and Mutases", Angewandte Chemie International Edition, 48(18), pp. 33623365, 2009. https://doi.org/10.1002/anie.200900337

[18] Datta, S., Christena, L. R., Rajaram, Y. R. S. "Enzyme immobilization: an overview on techniques and support materials", Biotech, 3(1), pp. 1-9, 2012. https://doi.org/10.1007/s13205-012-0071-7

[19] Ender, F. "Modeling, Realization and Characterization of Microreactors in Lab-on-a-Chip Device", PhD Thesis, Budapest University of Technology and Economics, 2016. [online], Available at: https:// repozitorium.omikk.bme.hu/bitstream/handle/10890/5320/ertekezes.pdf?sequence=2 [Accessed: 12 August 2018]

[20] Nayak, R., Padhye, R., Kyratzis, I. L., Truong, Y. B., Lyndon, A. "Recent advances in nanofiber fabrication techniques", Textile Research Journal, 82(2), pp. 129-147, 2011. https://doi.org/10.1177/0040517511424524

[21] Fogler, H. S. "Elements of Chemical Reaction Engineering", Prentice Hall, UK, 2008. 